\title{
AERODYNAMIC SHAPE OPTIMIZATION OF A PIPE USING THE ADJOINT METHOD
}

\author{
Eysteinn Helgason*, Siniša Krajnović \\ Division of Fluid Dynamics \\ Department of Applied Mechanics \\ Chalmers University of Technology \\ Gothenburg, Sweden \\ *Email: eysteinn@chalmers.se
}

\begin{abstract}
Shape optimization of an inlet pipe to an engine recirculator cooler using the adjoint method is presented. The method uses surface sensitivities calculated from an adjoint flow field implemented in the finite volume CFD solver OpenFOAM ${ }^{\circledR}$ [1]. This method allows for computation of the whole sensitivity field with only two solver calls, a primal and an adjoint solver call. A RANS solver with the standard k-epsilon turbulence model applying standard wall functions was used for the primal flow solver. The adjoint surface sensitivities are calculated from the adjoint and the primal flow fields and give information about how the objective function is affected by normal motion of the surface. The surface sensitivities are coupled to a mesh morphing library in OpenFOAM diffusing the motion of the boundary nodes to the internal cells of the mesh. The resulting geometry gave a $6.5 \%$ decrease in the total pressure drop through the pipe.
\end{abstract}

\section{NOMENCLATURE}

A Area of a cell face.

$J$ Cost function.

$k \quad$ Turbulence kinetic energy.

$n_{\alpha} \quad$ Number of design variables.

$p \quad$ Primal flow field pressure.

$q$ Adjoint pressure.

$\boldsymbol{u}$ Adjoint velocity.

$u_{n} \quad$ Normal component of the adjoint velocity.

$\boldsymbol{u}_{t} \quad$ Tangential component of the adjoint velocity. $v$ Primal flow field velocity.

$y^{+}$Dimensionless wall distance.

$\beta \quad$ Surface normal displacement.

$\varepsilon \quad$ Dissipation rate of the turbulence kinetic energy.

$v$ Kinematic viscosity.

$\rho$ Density.

\section{INTRODUCTION}

Optimization processes in CFD for industrial applications are governed by methods based on genetic algorithms that are in general computationally intensive. Another group of optimization methods based on sensitivity analysis is becoming an interesting choice for industrial applications. The group of sensitivity methods used in optimization can be divided into direct and adjoint methods. Direct sensitivity analysis methods are most efficient when obtaining sensitivities of many cost functions with respect to a few design variables as they are independent of the number of cost functions. An example of a direct difference method is the first order forward difference which needs $n_{\alpha}+1$ solver calls to provide the sensitivity map or in the case of central difference where the number of solver calls is $2 \cdot n_{\alpha}$, where $n_{\alpha}$ denotes the number of design variables. The adjoint method allows for these calculations using only two solver calls, one for the primal flow solver and one for the adjoint solver. This makes the adjoint method a feasible choice in the calculation of gradients for aerodynamic optimization in industrial applications as it is the most profitable one in terms of computational power. Here the number of design variables is generally much larger than the 
number of cost functions. The adjoint method is independent of the number of design variables while one adjoint solver call is generally needed for each cost function. This removes the constraint normally set by number of design variables, where now every cell in the flow domain can be described as a design variable without affecting the computational time. Sensitivity on the surface of the geometry can be derived from the adjoint and primal flow fields giving information on how normal motion of the surface of the geometry affects the cost function. This information can then be used for efficient fine tuning of an existing design.

The adjoint method for optimization gained popularity following the publication of Jameson [2] in 1988, where he applied the adjoint Euler equations to transonic 2D airfoils. He later used the adjoint Navier-Stokes equations to optimize a 3D wing [3]. For ducted flow, a simplification can be made to the adjoint equations as shown by Othmer [4]. This makes the continuous adjoint equations independent of the cost function which then only emerge in the boundary conditions for the adjoint solver.

The current work describes an optimization using the continuous adjoint method and surface sensitivities derived from the adjoint equations. The surface sensitivities are coupled to a mesh motion library in OpenFOAM that controls the deformation of the mesh. This method is then applied in an optimization loop, first to a relatively simple geometry where the flow is in the laminar region and then on a part of an inlet pipe to an engine recirculater cooler of a Scania truck. The boundary conditions of the inlet pipe resemble conditions in a truck at cruising speed, and the goal is to minimize the total pressure drop in the pipe.

\section{OPTIMIZATION USING THE ADJOINT METHOD}

This section describes the optimization process and the coupling of the mesh library with the adjoint surface sensitivities, beginning with a description of the adjoint equations and their connection to the adjoint surface sensitivities.

There are two main routes to follow in the implementation of the adjoint method. One is the so called discrete method where the flow equations are discretized, linearized and then adjointed. This method includes some partial derivatives that can either be implemented by manipulating the code manually, which requires considerable work, or be implemented using automatic differentiation tools that either automatically linearize and adjoint the computer code or assist in the manual differentiation work by applying them to individual routines. In the continuous implementation on the other hand, the adjoint equations are derived directly from the linearized flow equations. The adjoint equations are then discretized in the same manner as the primal flow field equations. Both of these methods have their advantages and drawbacks, which are discussed in more detail in [5] and [6]. In the derivation of the continuous adjoint equations it is common to assume "frozen turbulence", where the variation of the eddy viscosity, $v$, is neglected. Some work has been done on implementing adjoint turbulence models for the adjoint equations; see for example [7].

The current work makes use of the continuous implementation by Othmer et al. [8] in the open source CFD toolbox OpenFOAM. OpenFOAM is a non-staggered Finite Volume Method code written in $\mathrm{C}++$ using object oriented approach.

\section{Continuous Adjoint Method}

For the full derivation of the continuous adjoint equations readers are referred to a paper by Othmer [4]. Using the "frozen turbulence" assumption, where the variation of the eddy viscosity, $v$, is neglected, the general form of the adjoint incompressible Navier-Stokes equations is

$$
\begin{aligned}
-2 D(\boldsymbol{u}) \boldsymbol{v} & =-\nabla q+\nabla \cdot(2 v D(\boldsymbol{u}))-\frac{\partial J_{\Omega}}{\partial \boldsymbol{v}}, \\
\nabla \cdot \boldsymbol{u} & =\frac{\partial J_{\Omega}}{\partial p}
\end{aligned}
$$

where $D(\boldsymbol{u})=\frac{1}{2}\left(\nabla \boldsymbol{u}+(\nabla \boldsymbol{u})^{T}\right)$. The boundary conditions for the adjoint equations are shown in Eq. 2 .

$$
\begin{gathered}
\int_{\Gamma} \mathrm{d} \Gamma(\boldsymbol{n}(\boldsymbol{u} \cdot \boldsymbol{v})+\boldsymbol{u}(\boldsymbol{v} \cdot \boldsymbol{n}) \\
\left.+2 v \cdot D(\boldsymbol{u})-q \boldsymbol{n}+\frac{\partial J_{\Gamma}}{\partial \boldsymbol{v}}\right) \cdot \boldsymbol{\delta} \boldsymbol{v} \\
-\int_{\Gamma} \Gamma 2 \boldsymbol{v} \boldsymbol{n} \cdot D(\delta \boldsymbol{v}) \cdot \boldsymbol{u}=0, \\
\int_{\Gamma} \mathrm{d} \Gamma\left(\boldsymbol{u} \cdot \boldsymbol{n}+\frac{\partial J_{\Gamma}}{\partial p}\right) \delta p=0 .
\end{gathered}
$$

For the general form of the adjoint equations, both the adjoint equations and the boundary conditions depend on the cost function, $J$. This makes it necessary to modify both the boundary conditions and the adjoint equations for each cost function. For a cost function that is only dependent on the surface of the flow domain, as is generally the case for pipe flow, the adjoint Navier-Stokes equations can be simplified and are written out in Eq. 3, along with the boundary conditions for the wall and inlet in Eq. 4 and the boundary conditions for the outlet in Eq. 5.

$$
\begin{gathered}
\nabla \cdot \boldsymbol{u}=0 . \\
\boldsymbol{u}_{t}=0, \\
u_{n}=-\frac{\partial J_{\Gamma}}{\partial p}, \\
\boldsymbol{n} \cdot \nabla q=0 .
\end{gathered}
$$$$
-2 D(\boldsymbol{u}) \boldsymbol{v}=-\nabla q+\nabla \cdot(2 v D(\boldsymbol{u}))
$$ 


$$
\begin{aligned}
& q=\boldsymbol{u} \cdot \boldsymbol{v}+u_{n} v_{n}+\boldsymbol{v}(\boldsymbol{n} \cdot \nabla) u_{n}+\frac{\partial J_{\Gamma}}{\partial v_{n}} \\
& 0=v_{n} \boldsymbol{u}_{t}+\boldsymbol{v}(\boldsymbol{n} \cdot \nabla) \boldsymbol{u}_{t}+\frac{\partial J_{\Gamma}}{\partial \boldsymbol{v}_{t}}
\end{aligned}
$$

The goal in the optimization process is to minimize the total pressure drop through the pipe using the goal function

$$
J=\int_{\text {Inlet }} \mathrm{d} \Gamma c\left(p+\frac{1}{2} v^{2}\right)-\int_{\text {Outlet }} \mathrm{d} \Gamma c\left(p+\frac{1}{2} v^{2}\right)
$$

where $c=1 \mathrm{~m} / \mathrm{s}$ is added to compensate for missing units. ${ }^{1}$ Combining Eq. 6 with Eq. 4 gives us the boundary conditions for the adjoint velocity at the wall and the inlet as

$$
\begin{aligned}
& \boldsymbol{u}_{t}=0, \\
& u_{n}=\left\{\begin{array}{l}
0 \text { at the wall, } \\
-1 \mathrm{~m} / \mathrm{s} \text { at the inlet. }
\end{array}\right.
\end{aligned}
$$

The outlet boundary conditions are derived by inserting Eq. 6 into Eq. 5. The conditions for the adjoint pressure are given in Eq. 8 and the boundary condition for the tangential component of the adjoint velocity at the outlet in Eq. 9

$$
\begin{gathered}
q=\boldsymbol{u} \cdot \boldsymbol{v}+u_{n} v_{n}+\boldsymbol{v}(\boldsymbol{n} \cdot \nabla) u_{n}-v_{n}, \\
0=v_{n} \boldsymbol{u}_{t}+\boldsymbol{v}(\boldsymbol{n} \cdot \nabla) \boldsymbol{u}_{t}-\boldsymbol{v}_{t}
\end{gathered}
$$

The surface sensitivity used for the mesh deformation can be shown to be the normal gradient of the adjoint and primal velocities $[4,9,10]$,

$$
\frac{\partial L}{\partial \beta}=A v(\boldsymbol{n} \cdot \nabla) \boldsymbol{u}_{t} \cdot(\boldsymbol{n} \cdot \nabla) \boldsymbol{v}_{t}
$$

\section{Optimization Control and Mesh Deformation}

In the beginning, a converged solution of both the primal and adjoint flow fields is obtained. The adjoint and primal velocities are used to calculate the surface sensitivities at the beginning of the optimization process using Eq. 10. The sensitivities are smoothed and the mesh updated. The optimization loop starts again and the primal and adjoint flow solvers are solved until partial convergence is reached and the mesh updated again;

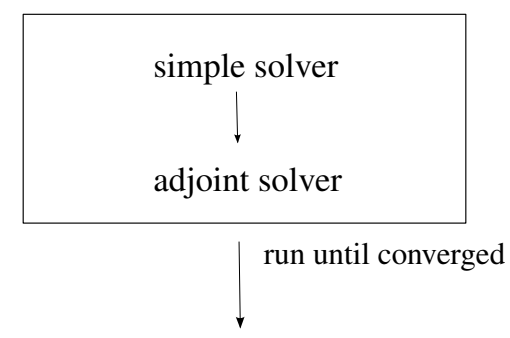

surface sensitivities

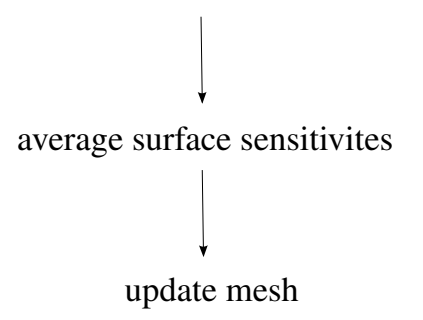

FIGURE 1: IN THE OPTIMIZATION LOOP THE PRIMAL AND ADJOINT FLOW SOLVERS ARE PARTIALLY CONVERGED BEFORE CALCULATING THE SURFACE SENSITIVITIES. AFTER UPDATING THE MESH THE LOOP STARTS AGAIN.

see Fig. 1. The loop continues until the surface sensitivities are below a certain criterion.

In this paper we employ a CAD-free method where all the surface sensitivities in the optimized region are used in the morphing process of the mesh. The mesh is morphed so that the nodes are either stretched or contracted and the simulation continues in the next iteration step with no removal or addition of cells. This saves computational time during the optimization process but can have a negative impact on the quality of the mesh.

The surface sensitivity is smoothed by interpolating the values from the face centers to points on each patch multiple times to smooth out the largest fluctuations. The smoothed sensitivities are then transformed into motion of the boundary nodes.

The motion of the internal nodes of the mesh is controlled by a mesh motion library in OpenFOAM that allows for the mesh motion of the boundary nodes to be diffused through the mesh. This diffusion of the mesh motion is governed by the Laplace smoothing equation with uniform diffusion,

$$
\nabla \cdot \nabla \boldsymbol{s}=0
$$

Here $\boldsymbol{s}$ is the mesh deformation velocity and the boundary conditions are the sensitivities that control the movement of the faces. The deformation of the boundary notes is controlled by a method of steepest decent, where the motion of the boundary is

\footnotetext{
${ }^{1}$ Correction made to the goal function compared to the original paper.
} 

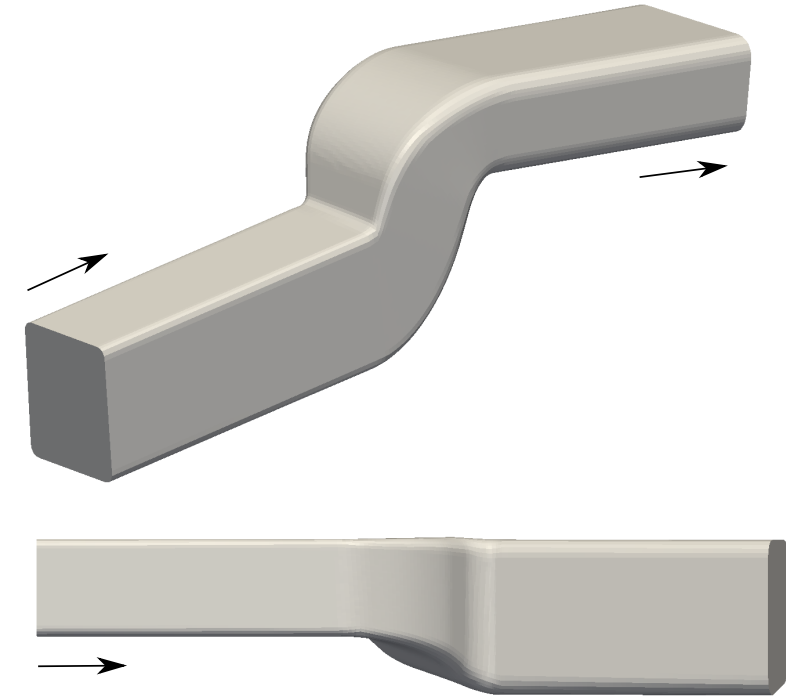

FIGURE 2: THE S-BEND SEEN FROM THE SIDE AND BELOW. THE FLOW DIRECTION IS FROM LEFT TO RIGHT AS DEPICTED BY THE ARROWS.

defined by:

$$
\boldsymbol{s}_{\Gamma}=\gamma \cdot \frac{\partial L}{\partial \beta},
$$

and $\partial L / \partial \beta$ is obtained from Eq. 10. The step size, $\gamma$, is based on the minimum face length of the mesh and normalized with the maximum initial surface sensitivity in the domain before any mesh update has been performed. This gives the largest mesh deformation at the beginning of the optimization process, and the deformations become smaller as the sensitivity on the surface decreases with each mesh update.

\section{APPLICATION}

Two geometries have been optimized, an S-bend with a laminar flow field and an inlet pipe to the exhaust gas re-circulator cooler of a Scania diesel engine. In the latter case the flow is turbulent, and turbulence modelling with wall functions was used for the primal flow solver.

\section{S-Bend}

This geometry is an S-shaped pipe consisting of three parts, inlet, outlet and walls; see Fig 2. The computational grid contains 225000 hexahedral cells. The inlet boundary conditions of the primal velocity are $v=1 \mathrm{~m} / \mathrm{s}$, and the kinematic viscosity, $v=1.6 \cdot 10^{-4} \mathrm{~m}^{2} / \mathrm{s}$, results in a laminar flow with a Reynolds number of 340 based on the hydraulic diameter of the pipe at the inlet. No slip is applied to the wall. For the adjoint solver, no slip and zero gradient for the adjoint velocity and pressure respectively are applied at the wall. The adjoint inlet and outlet boundary conditions are derived from the primal flow variables as described in Eqs. 7, 8 and 9. The whole wall section from the inlet to the outlet is selected for optimization with respect to total pressure drop.

\section{Inlet Pipe}

This optimization procedure has been applied to a real world engineering problem to emphasize the potentials of the method. Here follows a description of the case, the numerical parameters and the boundary conditions.

Geometry and Computational Grid. The geometry used in this optimization process is an inlet pipe to the exhaust gas re-circulator cooler of a Scania diesel engine. A part of the pipe has been selected for shape optimization, where the design will be fine tuned in order to minimize the pressure drop through the pipe; see Fig. 3. The inlet and the outlet of the pipe have been extended to avoid numerical problems.

The mesh used in the optimization process is a pure hex mesh created in ANSYS ICEM CFD containing around 1 million cells and having an average $y^{+}$value of 120 . The structure of the mesh at the inlet of the pipe is shown in Fig. 4. Two computational grids were used, containing 1.0 and 1.5 million cells. The same wall distance was applied to both grids while the resolution was higher further away from the walls for the finer mesh. The results gave a difference of less than $1.2 \%$ for the total pressure drop through the pipe for those two meshes. This difference in the results is found acceptable for the purpose of the present study, and the coarser computational grid used for the optimization.

Solver and Boundary Conditions. The solver applied for the primal flow is a steady state incompressible solver that uses the SIMPLE pressure correction. The standard k- $\varepsilon$ turbulence model [11] was used for the primal flow field along with standard wall functions. Simulations were performed with first order upwind discretization scheme for the convective fluxes. Various second order schemes were tested but did not give sufficient convergence for the adjoint solver and quickly resulted in a surface that was not sufficiently smooth. The boundary conditions applied to the primal flow equal those of a truck at cruising speed with inlet velocity to the pipe, $v$, equivalent to $40 \mathrm{~m} / \mathrm{s}$ and no slip condition for walls. Fixed value was used for pressure at the outlet and homogeneous Neumann boundary condition for inlet and walls. For the turbulence quantities, $k=24 \mathrm{~m}^{2} / \mathrm{s}^{2}$ and $\varepsilon=3943 \mathrm{~m}^{2} / \mathrm{s}^{3}$ at the inlet. The kinematic vis- 

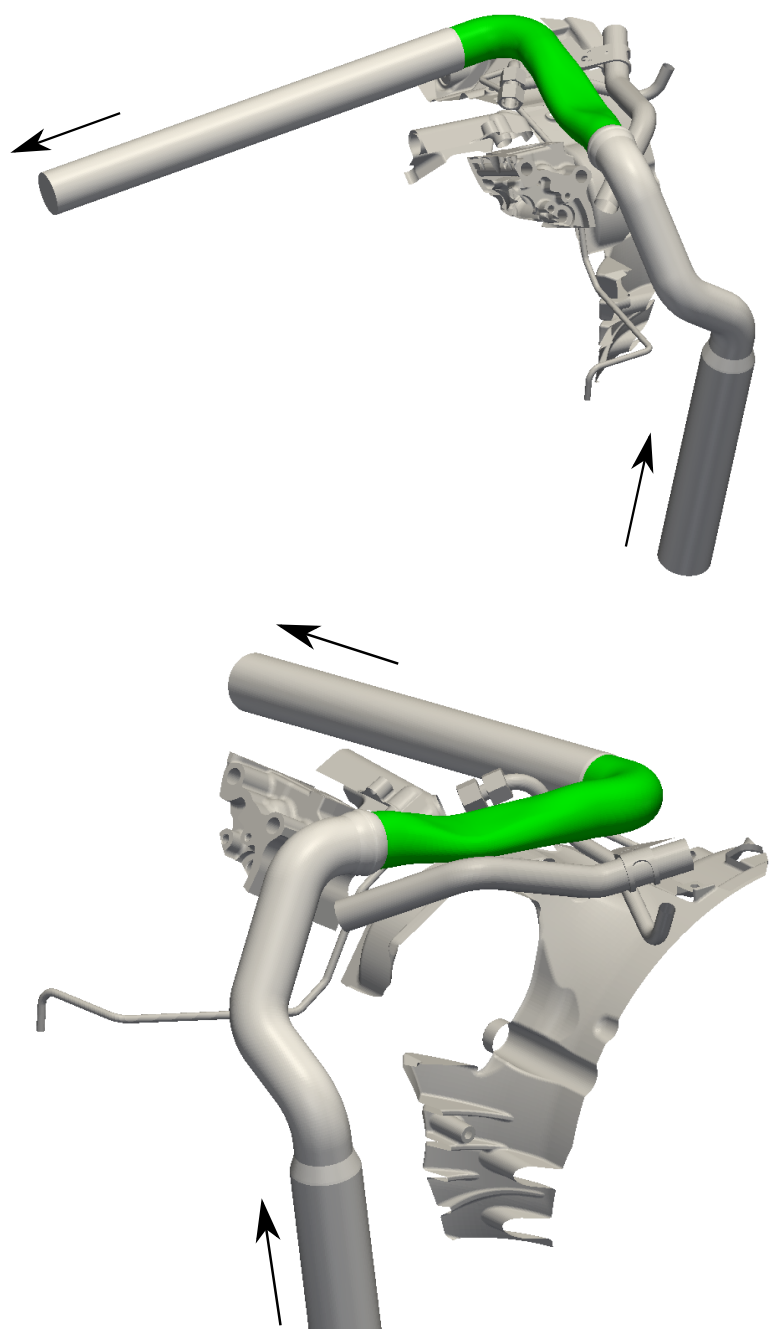

FIGURE 3: THE PIPE SHOWING IN GREEN THE AREA CHOSEN FOR OPTIMIZATION. THE INLET CAN BE SEEN AT THE BOTTOM OF THE FIGURES.

cosity, $v=1.0 \cdot 10^{-5} \mathrm{~m}^{2} / \mathrm{s}$, gives a Reynolds number of $1.9 \cdot 10^{5}$ based on the diameter of the pipe at the inlet.

The boundary conditions applied to the adjoint solver at the wall are no slip and homogeneous Neumann for the adjoint velocity and pressure, respectively. For the inlet and outlet the boundary conditions are derived from the primal flow variables as described in Eqs. 7, 8 and 9.

\section{RESULTS AND DISCUSSION}

This section shows the results from the optimization process and the effect on the objective function from each of the mesh updates.

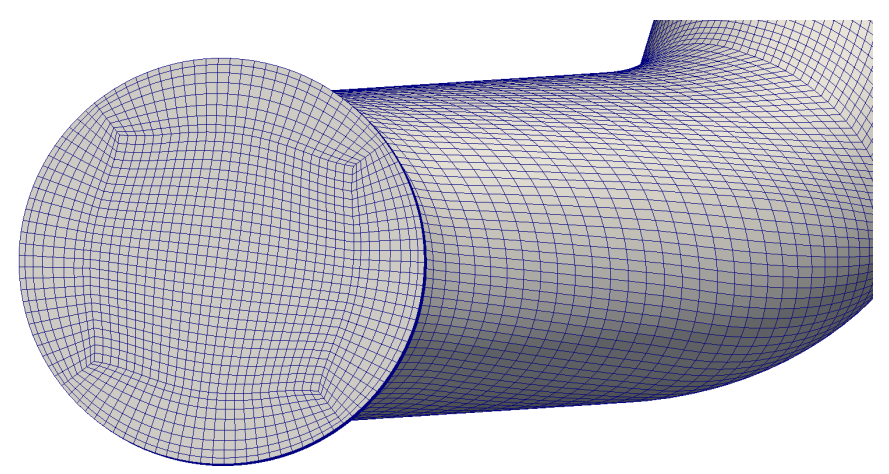

FIGURE 4: THE INLET OF THE STRUCTURED HEXAHEDRAL MESH.

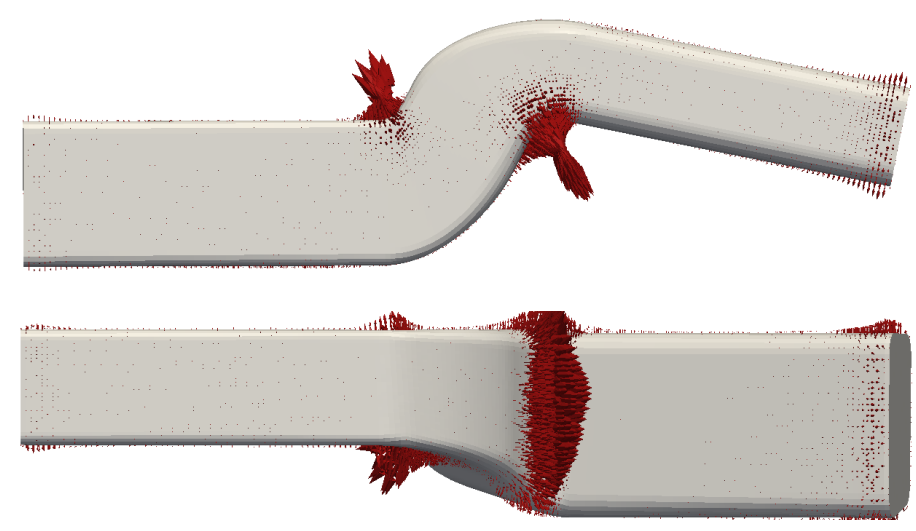

FIGURE 5: THE SURFACE SENSITIVITIES SHOW WHERE THE IMPACT ON THE OBJECTIVE FUNCTION FROM NORMAL MOTION OF THE SURFACE IS LARGEST.

\section{S-Bend}

The surface sensitivities were calculated for the whole pipe from the inlet to the outlet. This can cause problems in the cells closest to the boundaries, as the inlet and outlet patches are fixed and no motion at these points is allowed, which can decrease cell quality. In this case the largest sensitivities are close to the center of the domain, as is shown in Fig. 5, which shows the sensitivities on the surface for the original design. These sensitivities are used in the first deformation of the geometry.

Each of the geometries created is simulated until convergence, and the total pressure drop through the pipe is calculated. Figure 6 shows the effect from the geometry modifications on the total pressure after each mesh update. The largest deformation is in the first design change; this is when changes in the geometry have the largest impact on the objective function, as indicated by 


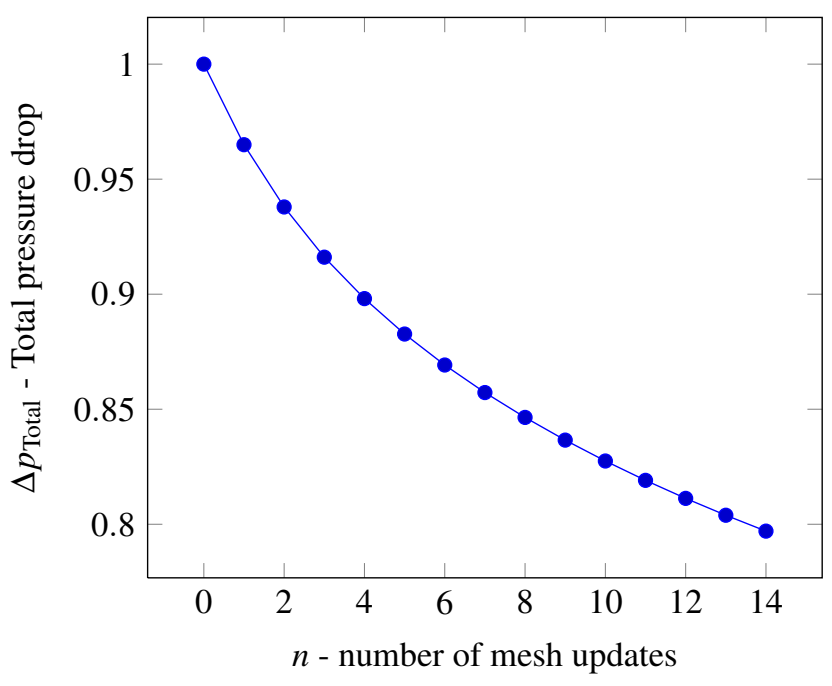

FIGURE 6: TOTAL PRESSURE DROP FOR EACH OF THE DESIGN UPDATES COMPARED TO THE ORIGINAL DESIGN FOR THE S-BEND. AFTER 14 MESH UPDATES THE TOTAL PRESSURE DROP HAD DECREASED BY $20 \%$.

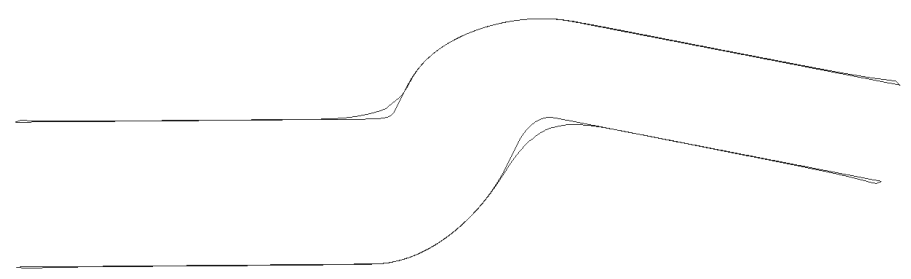

FIGURE 7: A CUT ALONG THE FLOW DIRECTION SHOWING THE CHANGES ON THE SURFACE OF THE BEND. THE OUTER LINE DENOTES THE OPTIMIZED GEOMETRY. THE LARGEST DEFORMATION IS AROUND THE BEND, SOME EXPANSION CAN ALSO BE NOTICED CLOSE TO THE OUTLET.

the surface sensitivities being largest for the original geometry compared to the subsequent geometries. The improved design showed a decrease of $20 \%$ in the total pressure drop through the pipe compared to the original geometry.

By comparing the original and optimized geometry, we see that, in the optimization process the pipe has a tendency to expand a little while the largest change is in the bend. This can be seen in Fig. 7, where the inner line denotes the original design and the outer line the optimized geometry.

Figure 8 shows the two geometries combined, where the dark region shows where the normal motion of the surface was outwards while the lighter region shows where the total motion of the surface was inwards, compared to the original geometry.
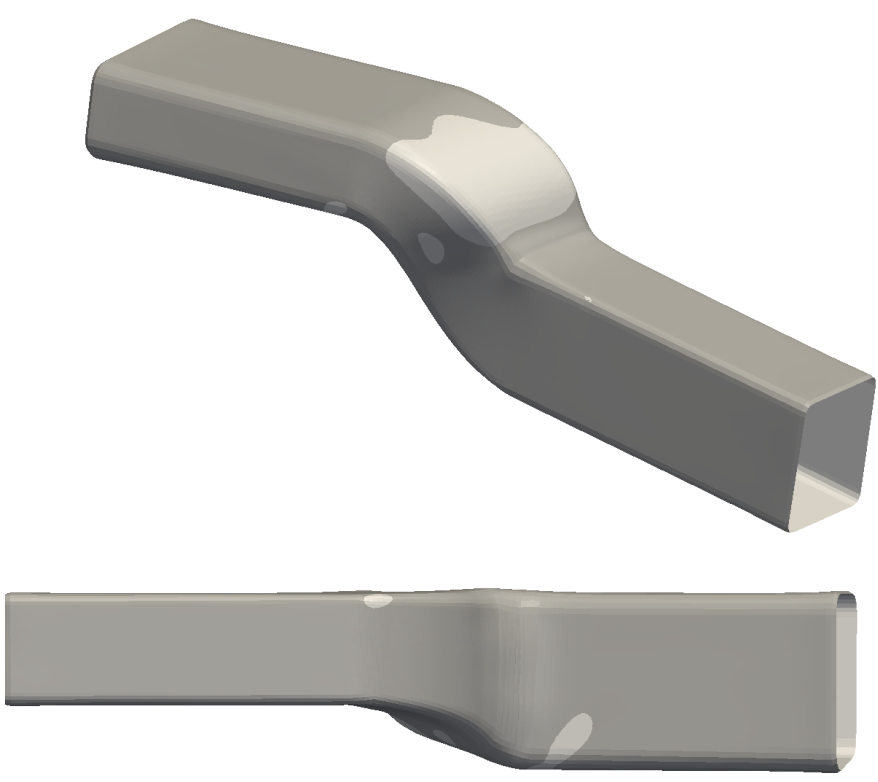

FIGURE 8: NORMAL MOTION OF THE SURFACE DURING THE OPTIMIZATION. THE DARK AND THE LIGHT COLORS DENOTE THE NORMAL MOTION OF THE SURFACE OUTWARD AND INWARD, RESPECTIVELY.

The whole optimization process took about four hours running on a single cpu on a desktop machine, or equivalent to ten primal flow simulations for the original geometry.

\section{Inlet Pipe}

The surface sensitivities from the original geometry show that the largest improvement in the objective function can be achieved by modifying the inside of the pipe just before the bend. Modification of this region gives the largest decrease in the goal function and therefore the most favorable design change. Figure 9 a shows the surface sensitivities on the original design before the optimization process started. The sensitivities on the surface are noticeably smaller after only ten updates of the geometry, as can be seen in Fig. 9 b.

A total of 35 mesh updates were performed. The largest modifications to the pipe were made at the beginning of the optimization in the region where the sensitivities were largest. In the optimization loop, the solvers are partially converged between each mesh update. However, to analyze the effects from the mesh deformation, each design was simulated until the primal flow solver had fully converged and the total pressure drop through the pipe was calculated. With each geometry, the magnitude of the surface sensitivity vectors usually decreases as effects from the design modification on the goal function decrease. The modifications to the geometry are directly coupled with the surface 


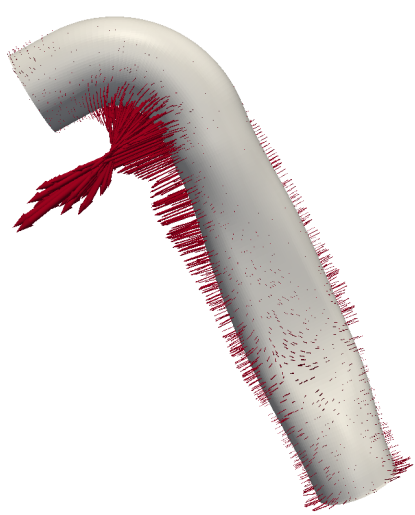

(a) Initial design. (b) After 10 mesh updates.

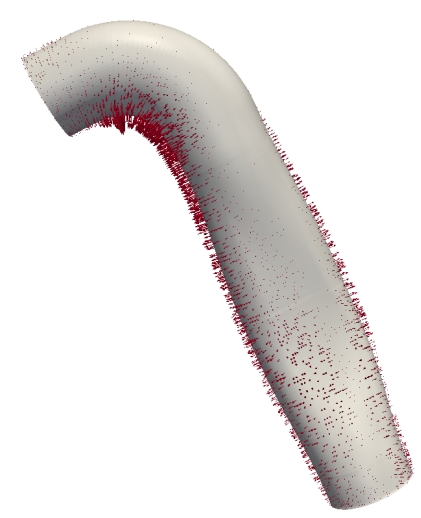

FIGURE 9: THE INITIAL SURFACE SENSITIVITIES COMPARED TO THE SENSITIVITIES AFTER 10 MESH UPDATES. OUTWARD MOVEMENT OF THE SURFACE ON THE INSIDE OF THE BEND GIVES THE LARGEST IMPROVEMENT OF THE DESIGN AT THE BEGINNING OF THE OPTIMIZATION PROCESS.

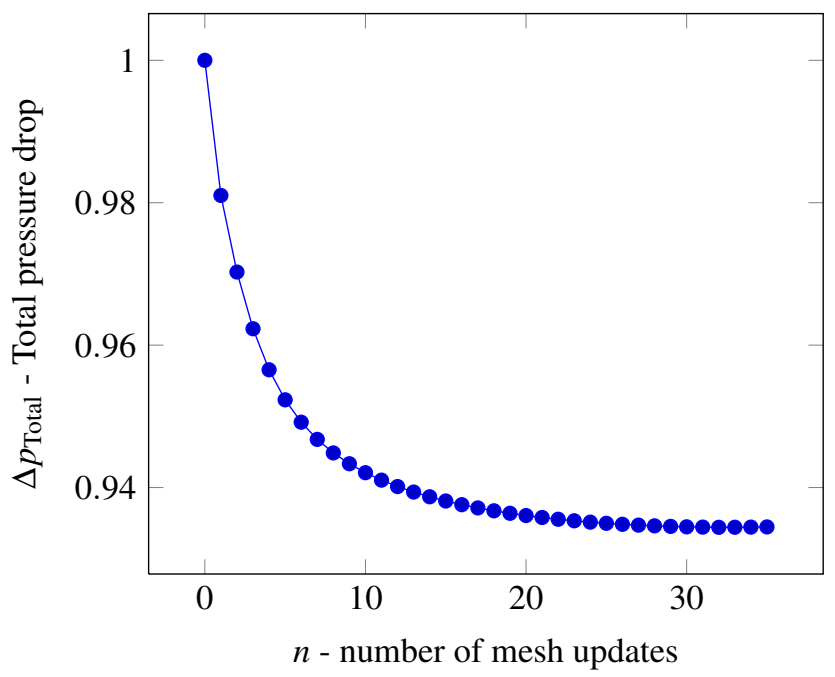

FIGURE 10: TOTAL PRESSURE DROP FOR THE WHOLE INLET PIPE. AFTER 35 MESH UPDATES THE TOTAL PRESSURE DROP HAD DECREASED BY 6.5\%.

sensitivities, and the modifications therefore become smaller and smaller. The total pressure drop through the pipe for each design compared to the original design can be seen in Fig. 10. The difference in the pressure drop between two successive designs decreases with each mesh update, as expected. After 35 optimization loops the total pressure drop through the whole pipe

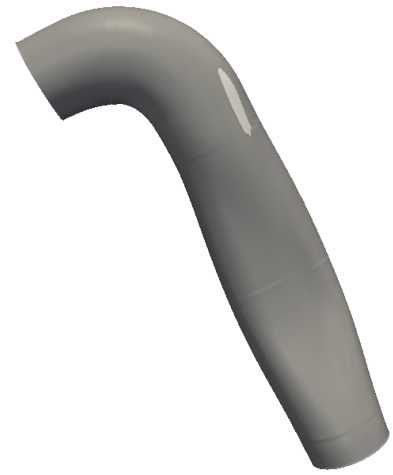

(a)

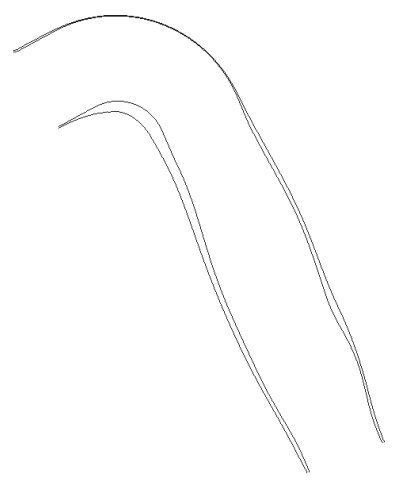

(b)
FIGURE 11: COMPARISON OF THE INITIAL AND OPTIMIZED DESIGN. IN FIGURE (a) THE DARK REGION DENOTES REGION OF EXPANSION OF THE PIPE WHILE THE LIGHT COLOR SHOWS A REGION WHERE THE SURFACE MOTION WAS INWARDS. FIGURE (b) SHOWS A CUT THROUGH THE PIPE WHERE THE OUTER LINE SHOWS THE FINAL DESIGN COMPARED TO THE INITAL. THE LARGEST DIFFERENCE BETWEEN THE TWO DESIGNS CAN BE SEEN ON THE INSIDE OF THE BEND.

has decreased by $6.5 \%$ compared to the original design.

Comparison of the final and initial designs shows the region of largest deformation on the inside of the pipe right before the bend where the surface sensitivities were largest at the beginning of the optimization process, Fig. 11. On the outside of the bend is a relatively small region where the total movement of the surface was inwards while the general tendency was to expand the pipe.

The run time for the whole optimization process involving the primal and adjoint flow solvers was roughly seven hours running in parallel on 16 processors, which is equivalent to six primal flow solver simulations for the original geometry.

As mentioned earlier, the simulations for both the primal and the adjoint solvers used first order upwind discretization scheme for both the primal and adjoint convective fluxes. This was necessary to obtain smooth surface sensitivities during the optimization procedure. To confirm that the optimized geometry gives a lower total pressure drop, both the initial and optimal geometries were simulated using a second order bounded Van Leer scheme for the convective fluxes. The results gave a $4.4 \%$ decrease in the total pressure drop between the two geometries.

Influence of the Shape Optimization on the Flow Figure 12 shows the influence of the geometry change on the pressure and velocity field in the center of the channel. The overall pressure in the optimized pipe, 12c, has increased compared 


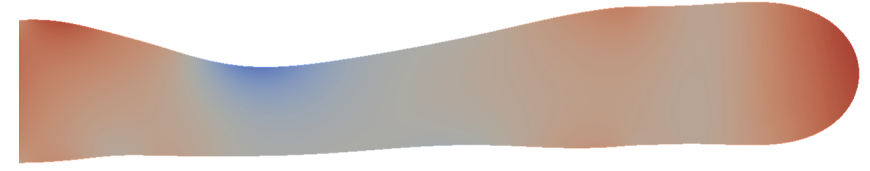

(a) Original geometry.

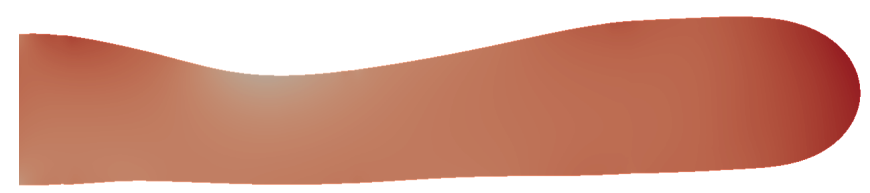

(b) Optimized geometry.

$$
p\left[\mathrm{~m}^{2} / \mathrm{s}^{2}\right] \underset{-2000}{-1000} 0,1000
$$

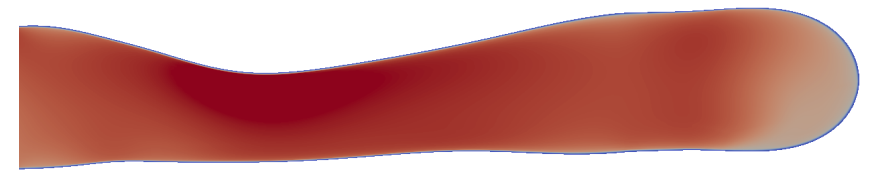

(c) Original geometry.

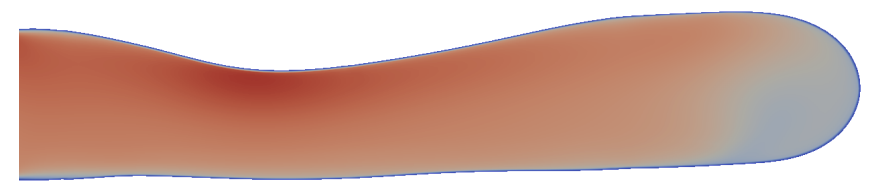

(d) Optimized geometry.
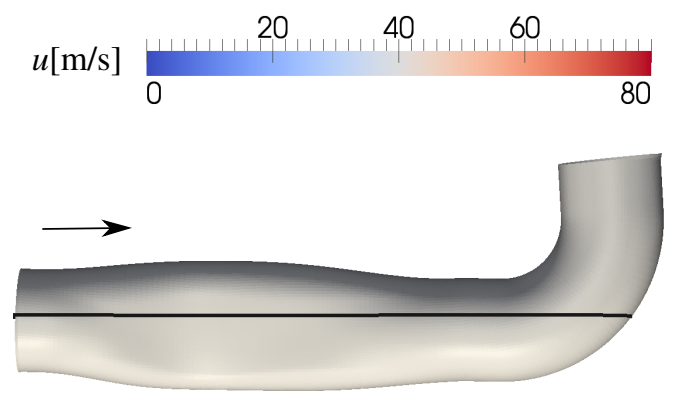

FIGURE 12: PLANE ALONG THE CENTER OF THE DEFORMED REGION SHOWING A COMPARISON OF PRESSURE, $p$, FOR THE ORIGINAL AND OPTIMIZED GEOMETRIES. THE LOCATIONS OF THE PLANES ARE SHOWN AS A BLACK LINE ON THE PIPE SURFACE.

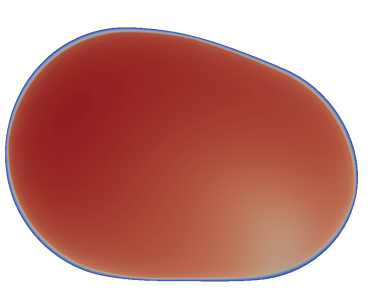

(a)

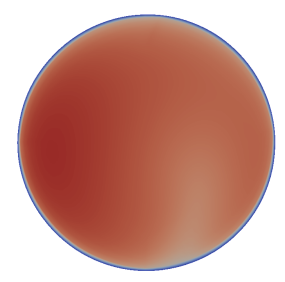

(c)

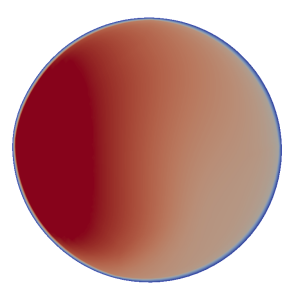

(e)

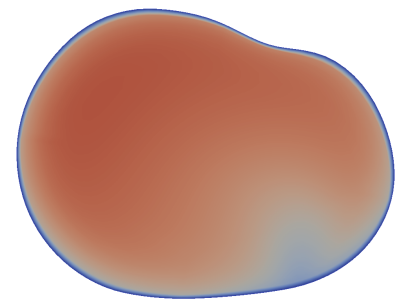

(b)

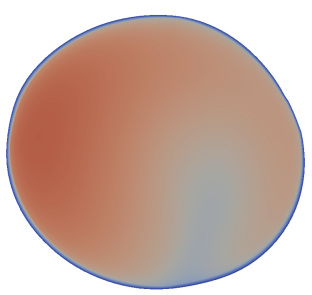

(d)

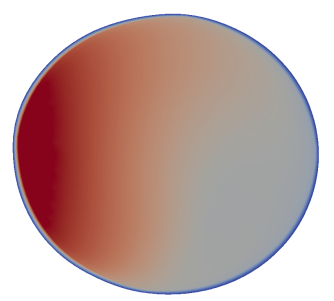

(f)
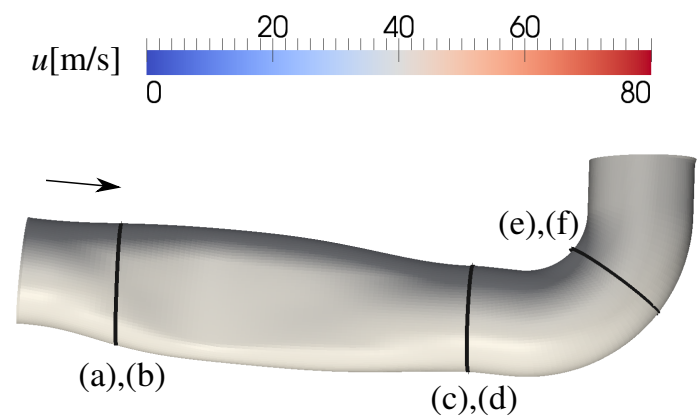

FIGURE 13: PLANES SHOWING COMPARISON OF MAGNITUDE OF VELOCITY, $u$, FOR THE ORIGINAL AND OPTIMIZED PIPES AT THREE DIFFERENT LOCATIONS. THE LOCATIONS OF THE PLANES ARE SHOWN AS BLACK LINES ON THE SURFACE. FIGURES (a), (c), AND (d) SHOW THE ORIGINAL GEOMETRY WHILE FIGURES (b), (e) AND (f) SHOW THE OPTIMIZED GEOMETRY. 
to the original geometry, Fig. 12b. The effect can also be observed in a lower velocity in the optimized pipe, Fig. 12d, compared to the original one in Fig. 12c. In Fig. 13, plane cuts taken at three different locations in the pipe show the effect on velocity magnitudes for the original and optimized geometry. The diameter of the pipe can be seen to expand for the optimized geometries compared to the original design.

\section{CONCLUSIONS}

Surface sensitivities obtained from primal and adjoint flow fields have successfully been applied in an optimization loop on an industrial geometry. The resulting geometry gave a $6.5 \%$ drop in the total pressure, which is significant value considering that only part of the pipe was selected for optimization. During this work the authors observed that, in order to obtain relatively smooth surface sensitivities, maintaining good mesh quality is of high importance. Automatic mesh generators, snappyHexMesh and FAME Hexa, were tested but resulted in noisy surface sensitivities, which after updating the mesh quickly resulted in a sawtooth shape on the surface of the geometry and poor quality cells. This makes the meshing process more time demanding than in commonly used optimization methods, while the accuracy and low demand for computational power gives this method an advantage. All flow solutions, both primal and adjoint, were performed using first order upwind for the convective fluxes. This is known to decrease the accuracy but was necessary in order to obtain sufficient convergence with the adjoint solver. The effect from the mesh updates on magnitude of the total pressure drop in the geometries is very different for the two cases, and it may appear that the improvement in the S-Bend is much larger. However, this can partly be explained by only a small part of the inlet pipe being selected for optimization while the total pressure drop is calculated through the whole pipe. It should also be noted that the step size is normalized with the largest surface sensitivity at the beginning of the optimization process, which causes the magnitude of the deformation to decrease faster for the inlet pipe than for the S-Bend.

\section{ACKNOWLEDGMENT}

This work was financially supported by the Swedish Energy Agency (sw. Energimyndigheten). The authors would like to acknowledge Scania AB for providing the inlet pipe geometry for this optimization process. Computations were performed at Swedish National Infrastructure for Computing (SNIC) at the Center for Scientific Computing at Chalmers (C3SE).

\section{REFERENCES}

[1] OpenFOAM ${ }^{\circledR}$ open source CFD toolbox. The OpenFOAM Foundation homepage: http://openfoam.com [2011].

[2] Jameson, A., 1988. "Aerodynamic design via control theory”. Journal of Scientific Computing, 3(3), pp. 233-260.

[3] Jameson, A., and Martinelli, L., 1998. "Optimum Aerodynamic Design Using the Navier Stokes Equations". Theoretical and Computational Fluid Dynamics, 10, pp. 213237.

[4] Othmer, C., 2008. "A continuous adjoint formulation for the computation of topological and surface sensitivities of ducted flows". International Journal for Numerical Methods in Fluids, 58, pp. 861-877.

[5] Peter, J. E., and Dwight, R. P., 2010. "Numerical sensitivity analysis for aerodynamic optimization: A survey of approaches". Computers \& Fluids, 39, pp. 373-391.

[6] Giles, M. B., and Pierce, N. A., 2000. "An Introduction to the Adjoint Approach to Design". Flow, Turbulence and Combustion, 65, pp. 393-415.

[7] Zymaris, A., Papadimitriou, D., Giannakoglou, K., and Othmer, C., 2010. "Adjoint wall functions: A new concept for use in aerodynamic shape optimization". Journal of Computational Physics, 229, pp. 5228-5245.

[8] Othmer, C., Villiers, E. d., and Weller, H. G., 2007. "Implementation of a continuous adjoint for topology optimization of ducted flows". AIAA 2007-3947.

[9] Pironneau, O., 1984. "Optimal Shape Design for Elliptic Systems”. Springer-Verlag.

[10] Soto, O., and Löhner, R., 2004. "On the Computation of Flow Sensitivities From Boundary Integrals". AIAA-040112.

[11] Launder, B. E., and Spalding, B. I., 1974. "The Numerical Computation of Turbulent Flows". Computer Methods in Applied Mechanics and Engineering, 3, pp. 269-289. 